\title{
Design of Fermentation Process Monitoring and Control System Based on LabVIEW
}

\author{
Feng Guo \\ College of Control Engineering, Northeastern University \\ in Qinhuangdao, Hebei, China \\ E-mail: 18712779923@163.com
}

\author{
Dong-Shuai Cui \\ Project Management, State Grid Aohan County Power \\ Supply Company, Chifeng, Inner Mongolia, China \\ E-mail:zdh2568069@163.com
}

\author{
Jie Li, Jia-Yu Cui, Chun-Tao Zhang \\ College of Control Engineering, Northeastern University in Qinhuangdao, Hebei, China \\ E-mail:18712779923@163.com
}

\begin{abstract}
This paper focuses on the commonly used glass fermentation tank in biological fermentation, aiming at improving and optimizing the current monitoring process of biological fermentation. Based on the LabVIEW platform, we have designed a set of monitoring system to study biological fermentation process. In order to realize the connection of the hardware part of the circuit, the field data acquisition signal is transmitted to the host computer through the data acquisition card, and to design and develop the monitoring and control interface through LabVIEW. Through the interaction between the upper computer and the lower computer, a set of intelligent automatic control monitoring system is built. System communication consists of the small system, which is composed of data acquisition card to gather and transform temperature, pH value and other signals. The data is collected and transmitted to the computer by using the PCI-6221 NI data acquisition card, then the data are processed and analyzed, The signal is stored and is displayed under the LabVIEW development platform. In the end, the control interface of PC displays the parameter of dissolved oxygen, $\mathrm{pH}$ value and temperature in the real-time fermentation environment. The whole fermentation process to achieve on-line detection, and realizes the preliminary foundation for the system real-time control.
\end{abstract}

\section{Keywords-fermentation; LabVIEW; data collection; monitoring wireless networks}

\section{INTRODUCTION}

Microbial fermentation technology is the basis of biochemical engineering modern biotechnology and technology industrial ${ }^{[1]}$. The fermentation process is the process of using microorganisms life activities in aerobic or anaerobic conditions to reproduce microbial body itself or preparation metabolites and secondary metabolites. Through the fermentation process we can acquire many humans which can't or is difficult to obtain synthetic material by other means. The fermentation process control technology is to control the temperature, $\mathrm{PH}$ and related environmental parameters in order to improve the process of biological reaction by using related technical means, for the whole of the fermentation process experiment or production, the fermentation process control technology is very important.
Our Country has greatly left behind the developed countries or advanced technology of other countries in fermentation process control technology level. That mainly embodies in the level of automation overall, in which the fermentation process is not high, the control is not accurate, even some fermented workshop still use the traditional control method, the operating personnel can achieve nutritional material adding or adjustment of control parameters in fermentation process only by experience However, the microorganism fermentation is the biological chemical reaction process, with nonlinear and lag and other characteristics, it is difficult to build the accurate mathematical model for fermentation process, and because of the current technology development restriction, some can only reflect the main parameters of fermentation process, but cannot be directly measured online, which makes the control process more complicated ${ }^{[2]}$.

In recent years, with the rapid development in microelectronic technology, detection technology, automatic control technology and computer technology, microbial fermentation process control will also develop towards in the respect of automation, digitization, intellectualization, it is possible to analyze and control the complex fermentation process. Gregory and Keay ${ }^{[3]}$ have already used LabVIEW to set up a system which controls the growth rate of yeast in view of the yeast feed batch culture development in early stage. Currently LabVIEW is the most widely used, the fastest developing, the most powerful graphical virtual instrument development tools. It abandons the texts and uses icons to programming, which is a graphical programming language, its more intuitive and easier to be accepted. The monitoring and control system which based on virtual instrument has excellent performance, strong expansibility and easy integration. The fuzzy algorithm ${ }^{4]}$, BP neural network $^{[5]}$, support vector machine ${ }^{[6]}$, ant colony algorithm ${ }^{[7]}$ and other nonlinear intelligent control algorithms can be easily applied to the control method of fermentation process. Because the domestic traditional brewing industry and the modern fermentation industry are more labor-intensive industries, the degree of automation is still relatively low. Through the design and optimization of fermentation process monitoring control system, it can realize effective monitoring and control of fermentation process, ensure efficient 
fermentation production process quality and improve the level of output of fermentation technology and process of quality control, obviously it has very important practical significance. Thus in this paper, the biological fermentation process monitoring system which based on LabVIEW platform is designed and developed in order to meets those needs and also change the technical situation. As for the common glass fermentation tank in the enterprise, it can achieve the function of the fermentation process monitoring, which lays the foundation for intelligent algorithm control in the future.

\section{GENERAL SYSTEM FORMULA}

A fermentation monitoring platform needs not only hardware devices, but also a well run, performing well, excellent software. With the rapid development of computer technology, the successful design of the various sensors, and constant improvement of the performance, the fermentation process online monitoring technology is increasingly maturing, and developing toward multiple parameters, high precision and automatic direction. This system uses LabVIEW as the development software of host computer fermentation process monitor and control system, designing a real-time monitoring system that can realize on-line detection of the whole process of fermentation, in which SHC68-68 - EPM Cable connect the junction box that accept sensor signals with NI PCI - 6221 connection, this data acquisition card system realize acquisition, conditioning and conversion of temperature, $\mathrm{PH}$ value, dissolved oxygen and other signal data, and then to transfer the data to the computer, with the LabVIEW development platform, in the LabVIEW development platform, data Various processing, analysis, and signal storage, display, the software part adopts the module and hierarchical idea, design LabVIEW program from up to down. System can collect data regularly, and save analysis and process the data, the fermentation monitoring system design principle is shown in figure 1.

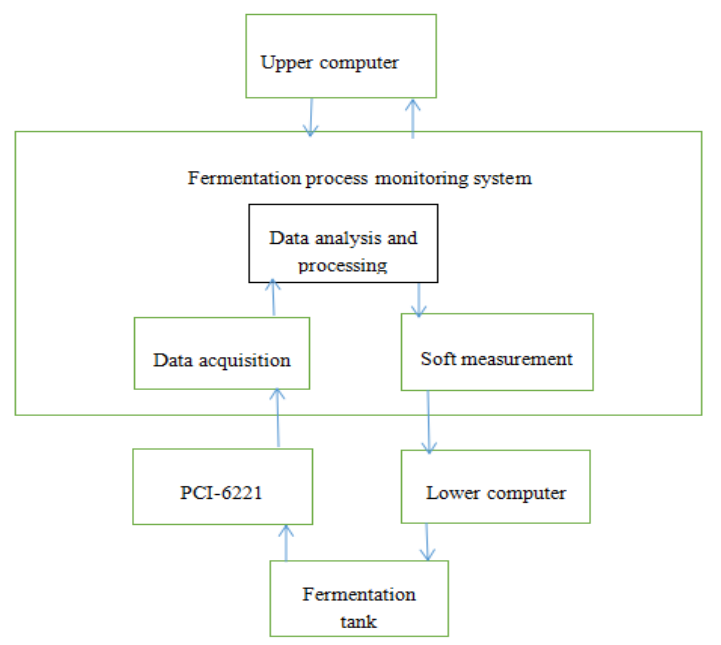

Figure 1. Fermentation monitoring system design principle diagram

In this systemthe peripheral equipment is mainly divided into two parts. One of them is a sensor or other measuring device, the other one is a signal acquisition device. The PCI6221 is used as the key data acquisition equipment, which is in charge of the analog/digital signal conversion. The computer programming is realized by using DAQ acquisition channel, it can save the scene-collected signal after $\mathrm{A} / \mathrm{D}$ conversion in the internal buffer.PCI-6221 adopts more circular manner in the long time high-speed data acquisition. In this way, it can start a collection process for countless times A/D conversion. A NI-DAQmx virtual channel includes a DAQ device of physical channel and configuration information of the physical channel. Such as the input range and custom scaling. The task of a NIDAQmx is virtual channel, timing, and trigger the information, as well as other related to the acquisition or generated attributes combination. The acquisition parameters are set to meet the fermentation process and equipment conditions. Only channel, set sampling parameters are chosen can the DAQmx further drive after work, and grouping samples collected. Sampling principle of channel of the process, as shown in figure 2 .

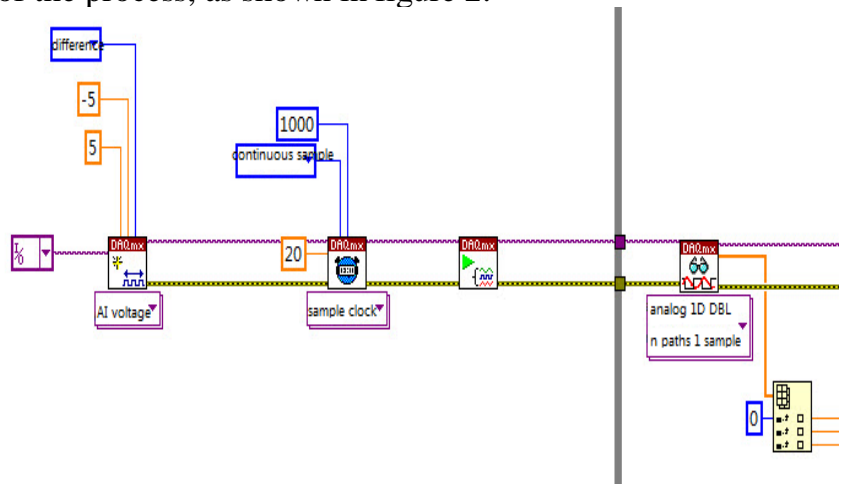

Figure 2. Sampling channel of the process principle diagram

But because the data acquisition card is a little expensive, that many laboratories are not equipped with the corresponding data acquisition card, we can use wireless sensor network to replace the data acquisition card to complete the data collection at this time. Wireless sensor networks have the advantage of distributed multi-point measurement, which can display and store the measured data, and the serial port receiving interrupt program can be applied to other serial port data acquisition system, it has a strong advantage of portability, which is also a good way of data collection.

\section{DESIGN OF HOST COMPUTER MONITORING SYSTEM THROUGH LABVIEW}

\section{A. Software Modular Function Design}

According to reality conditions of the commonly used glass fermentation tank of biological fermentation and the programming ideas of LabVIEW, the whole host computer is divided into several modules, and then integrating them by calling the subroutines. Due to the microorganism fermentation is in need of a stable living environment, in order to meet the control requirements, the whole system form a feedback loop control, controlling the oxygenthe $\mathrm{PH}$ 
and the temperature. At the same time, we can control each parameter values change to provide security environment for fermentation by controlling the Mixing motor speedthe $\mathrm{PH}$ and the Fermentation tank base heater.

Due to the particularity of fermentation experiment and high sensitivity to the fermentation environment it is essential to ensure the stable operation and good safety system, and it also requires the system to set the transfinite alarm function. If fermentation conditions have larger fluctuations and beyond the cordon, the system will automatically alarm and the alarm data will be stored in the databaseand it can improve the establishment of the expert system and enhance the value of historical data query. And the $\mathrm{PH}$ value alarm function design as shown in figure 3.

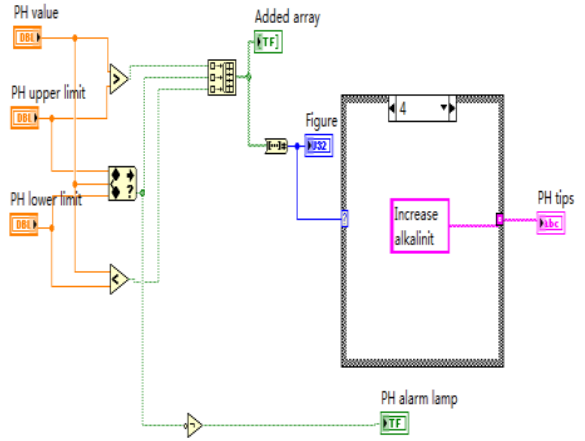

Figure 3. PH alarm design program

Besides meeting the real-time monitoring of fermentation temperature, $\mathrm{pH}$ value, oxygen value and stirring motor speed, the system also needs to save the real-time data in order to achieve data display, processing, analysis and use, which has a decisive sole for historical data inquire and establishment of expert system. The principle of the program design is shown in figure 4.

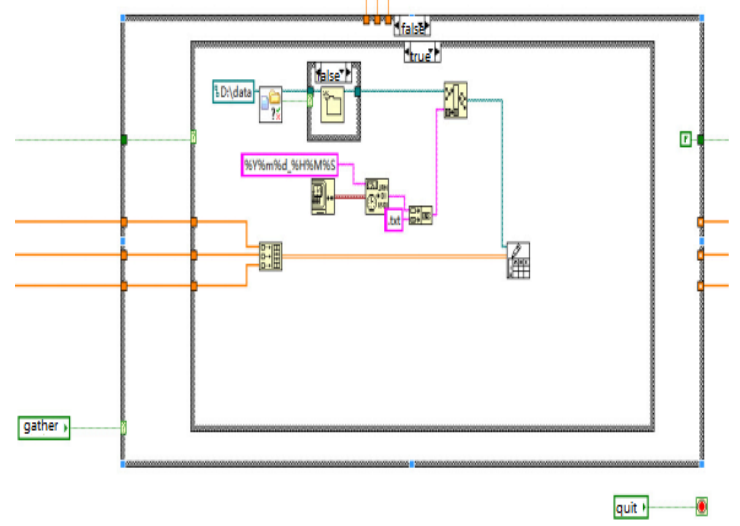

Figure 4. Data save and call program

\section{B. Main Page Display of Monitoring Control System}

Designing the monitoring master system can reflect the fermentation process and sate intuitively and automatic modulation control through LabVIEW's graphical visual interface editor, according to the original control system parameters as the reference value, comparing the collected data with the reference value to determine whether the system display is accurate and reliable or not, how much time will the system delay and how much influence will the control make. Through a series of debugging, which enables the system to achieve a good monitoring and control effect, realizing the intuitive display of system. The interface part function design of realizing real-time $\mathrm{pH}$ value collection and control among that is shown in figure 5.

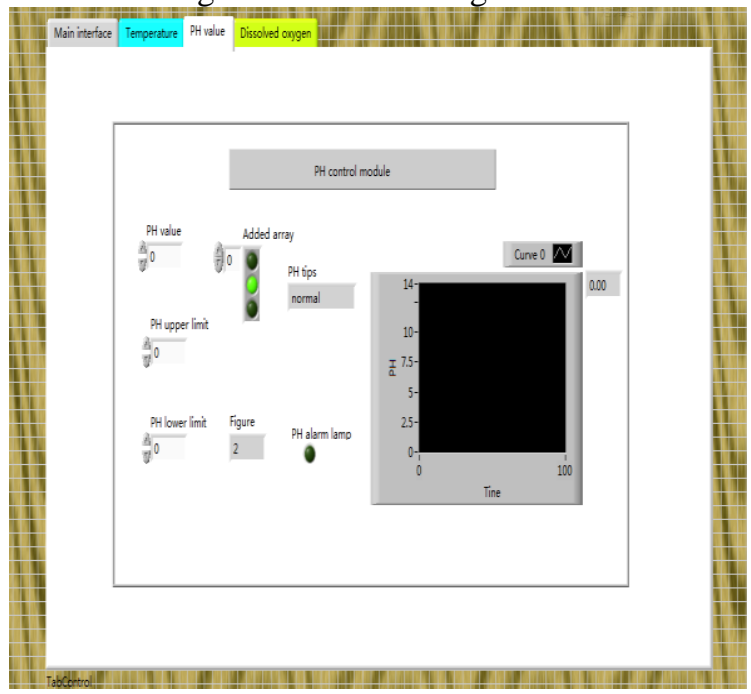

Figure 5. Design of real-time $\mathrm{PH}$ data acquisition interface

\section{CONCLUSION}

By studying the parameters influencing the fermentation process, we have designed a set of biological fermentation process monitoring system which is based on the LabVIEW platform. In the LabVIEW development platform, via multiple processing and analysis of data, the signal is stored and displayed by the PC display monitor interface, oxygen, $\mathrm{pH}$ and temperature of fermentation environment real-time measurement value, realizing the online detection of the whole fermentation process, through field experiment, the fermentation monitoring system can be effectively carried out data acquisition and analysis, verifying the effectiveness of the system, do the preliminary foundation for realizing real-time control system. LabVIEW can also be easily connected to the Internet, and also can be linked through the wireless sensor network, as a result it can achieve further distance online monitoring and control.

\section{ACKNOWLEDGMENT}

This research was supported by the basic scientific research service of Northeastern University (N142303014), Science and technology research project of Hebei Province (Z2014085) Scientific research foundation of Northeastern University at Qinhuangdao (XNB201411), Science and technology innovation project of Northeastern University at Qinhuangdao. 


\section{REFERENCES}

[1] R.J. Seviour, L.M. Harvey, M. Fazenda, et al. 5 - Production of foods and food components by microbial fermentation: an introduction[J]. Microbial Production of Food Ingredients, Enzymes and Nutraceuticals, 2013: 97-124.

[2] Zou Youfeng. Research on the Control System for Process of Glutamate Fermentation [D]. Lanzhou University of Technology, 2012.

[3] M.E. Gregory, P.J. Keay, P. Dean, et al. A Visual programming genvironment for bioprocess control[J]. Journal of Biotechnology, 1994, 33(3): 233-241.

[4] G. Tairidis, G. Foutsitzi, P. Koutsianitis, et al. Fine tuning of a fuzzy controller for vibration suppression of smart plates using genetic
algorithms[J]. Advances in Engineering Software, 2016, 101: 123135.

[5] Bing Wu, Shaojun Han, Jin Xiao, et al. Error compensation based on BP neural network for airborne laser ranging[J]. Optik - International Journal for Light and Electron Optics, 2016, 127(8): 4083-4088.

[6] Jian-Xun Peng, Karen Rafferty, Stuart Ferguson. Building support vector machines in the context of regularized least squares[J]. Neurocomputing, 211(26) 2016: 129-142.

[7] Youchuan Wan, Mingwei Wang, Zhiwei Ye, et al. A feature selection method based on modified binary coded ant colony optimization algorithm[J].Applied Soft Computing, 2016, 49: 248-258. 